\title{
Accelerating Knee MRI: 3D Modulated Flip-Angle Technique in Refocused Imaging with an Extended Echo Train and Compressed Sensing
}

\author{
He Sui ${ }^{1} *$, Jin Li ${ }^{2,3, *}$, Lin Liu', Zhongwen Lv', Yunfei Zhang ${ }^{4}$, Yongming Dai ${ }^{4}$, Zhanhao Mo' \\ 'China-Japan Union Hospital of jilin University, Changchun, People's Republic of China; ${ }^{2}$ jilin Province People's Hospital, Changchun, People's Republic \\ of China; ${ }^{3}$ The Department of Trauma Surgery, Shanghai Oriental Hospital, Shanghai, People's Republic of China; ${ }^{4}$ Central Research Institute, United \\ Imaging Healthcare, Shanghai, 20I800, People's Republic of China \\ *These authors contributed equally to this work \\ Correspondence: Zhanhao Mo, China-Japan Union Hospital of jilin University, No. I26 Xiantai Street, Erdao District, Changchun, People's Republic of \\ China, Email mozhanhao@jlu.edu.cn
}

Purpose: The three-dimensional (3D) sequence of magnetic resonance imaging (MRI) plays a critical role in the imaging of musculoskeletal joints; however, its long acquisition time limits its clinical application. In such conditions, compressed sensing (CS) is introduced to accelerate MRI in clinical practice. We aimed to investigate the feasibility of an isotropic 3D variable-flip-angle fast spin echo (FSE) sequence with CS technique (CS-MATRIX) compared to conventional 2D sequences in knee imaging.

Methods: Images from different sequences of both the accelerated CS-MATRIX and the corresponding conventional acquisitions were prospectively analyzed and compared. The signal-to-noise ratio (SNR) and contrast-to-noise ratio (CNR) of the structures within the knees were measured for quantitative analysis. The subjective image quality and diagnostic agreement were compared between CS-MATRIX and conventional 2D sequences. Quantitative and subjective image quality scores were statistically analyzed with the paired $t$-test and Wilcoxon signed-rank test, respectively. Diagnostic agreements of knee substructure were assessed using Cohen's weighted kappa statistic.

Results: For quantitative analysis, images from the CS-MATRIX sequence showed a significantly higher SNR than T2-fs 2D sequences for visualizing cartilage, menisci, and ligaments, as well as a higher SNR than proton density (pd) 2D sequences for visualizing menisci and ligaments. There was no significant difference between CS-MATRIX and 2D T2-fs sequences in subjective image quality assessment. The diagnostic agreement was rated as moderate to very good between CS-MATRIX and 2D sequences.

Conclusion: This study demonstrates the feasibility and clinical potential of the CS-MATRIX sequence technique for detecting knee lesions The CS-MATRIX sequence allows for faster knee imaging than conventional 2D sequences, yielding similar image quality to $2 \mathrm{D}$ sequences.

Keywords: knee MRI, compressed sensing, 3D variable-flip-angle fast spin echo

\section{Introduction}

As a crucial weight-bearing joint, the knee can easily sustain a variety of injuries, including meniscus tears, crucial ligament tears, and cartilage and bone injuries. Nevertheless, it is especially challenging to evaluate knee imaging compared with other musculoskeletal joints because of the knee's complex anatomical structures, which require equally high in-plane resolution and slice thickness to depict the anatomical structures clearly and to identify varying degrees of injuries, especially tears of the menisci and crucial ligaments. ${ }^{1,2}$

Magnetic resonance imaging (MRI) is considered to be a non-invasive technique in musculoskeletal imaging, because of its reliability, safety, and other advantages over diagnostic arthroscopy. ${ }^{3,4}$ Conventional two-dimensional (2D) sequences have been widely used in evaluating musculoskeletal injuries; however, their inherent disadvantages are widely reported, including low resolution along the slice selection direction, signal interference between contiguous 
slices, and blood flow artifacts. Isotropic three-dimensional (3D) sequences, which allow for higher spatial resolution and signal-to-noise ratio (SNR), have been demonstrated to have similar diagnostic performance to conventional 2D sequences on lesions in musculoskeletal joints. ${ }^{2,5-8}$ With a single 3D isotropic or near-isotropic acquisition, multiplanar reconstruction (MPR) can be carried out, which is especially beneficial for the evaluation of menisci, ligaments, tendons, and nerves. ${ }^{9-12}$ Nevertheless, the long acquisition time of 3D sequences limits their routine clinical use. ${ }^{13}$

The latest advances in MRI acceleration technology are contributing toward the advancement of 3D knee MRI. Rapid imaging techniques in musculoskeletal imaging have been introduced, such as compressed sensing (CS), based on a novel mathematical framework, providing higher data undersampling in k-space with nonlinear reconstruction, as well as improvements in imaging quality. ${ }^{14}$ This technique is based on the same principles used in digital image compression algorithms, which exploit inherent sparsity (lack of information) transformations on mathematical image data to reduce digital image size while maintaining perceived quality. Like parallel imaging techniques, CS techniques are more suited for 3D sequences that inherently have greater data sparsity than 2D sequences, and have been successfully implemented in musculoskeletal MRI. ${ }^{14-16}$ However, the application of CS in the 3D fast-spin-echo (FSE) sequence with true voxel isotropy is still limited, and the 3D FSE sequence with CS is confined to scanners from specific vendors. ${ }^{17}$ In view of the aforementioned points, we aim to apply a 3D modulated flip-angle technique for knee imaging, which is an FSE-based sequence with variable flip angles in refocused imaging with an extended echo-train (MATRIX) sequence. The MATRIX sequence can also accelerate imaging by using longer echo train lengths (ETLs) and shorter echo spacing (ESP) than a traditional FSE sequence. ${ }^{18}$

In this study, the 3D MATRIX sequence combined with a CS technique (CS-MATRIX) was employed to evaluate knee imaging. As the conventional 2D sequence serves as a well-accepted method used in routine diagnosis, this study was designed to investigate the feasibility of 3D CS-MATRIX sequences in knee imaging compared to conventional 2D sequences, regarding their image quality and diagnostic performance, and the acquisition time.

\section{Methods}

\section{Study Objectives and Case Selection}

This study was approved by the Institutional Review Board (IRB) of China-Japan Union Hospital of Jilin University. All procedures performed in this study involving human participants were in accordance with the ethical standards of the institutional and/or national research committee and with the 1964 Declaration of Helsinki and its later amendments or comparable ethical standards. The experimental flowchart is shown in Figure 1. We included 74 knees (from 69 symptomatic patients) enrolled in our department between February and August 2019 for MRI due to knee pain. The exclusion criteria included contraindications for MRI (eg, pacemakers, non-MRI-compatible metallic implants), previous knee surgery, tumor, infection, pediatric patients under the age of 18 , being unable to complete the inspection during the examination, and those whose images had serious artifacts after examination. As a result, 68 knees (from 63 symptomatic patients, male: 33, mean

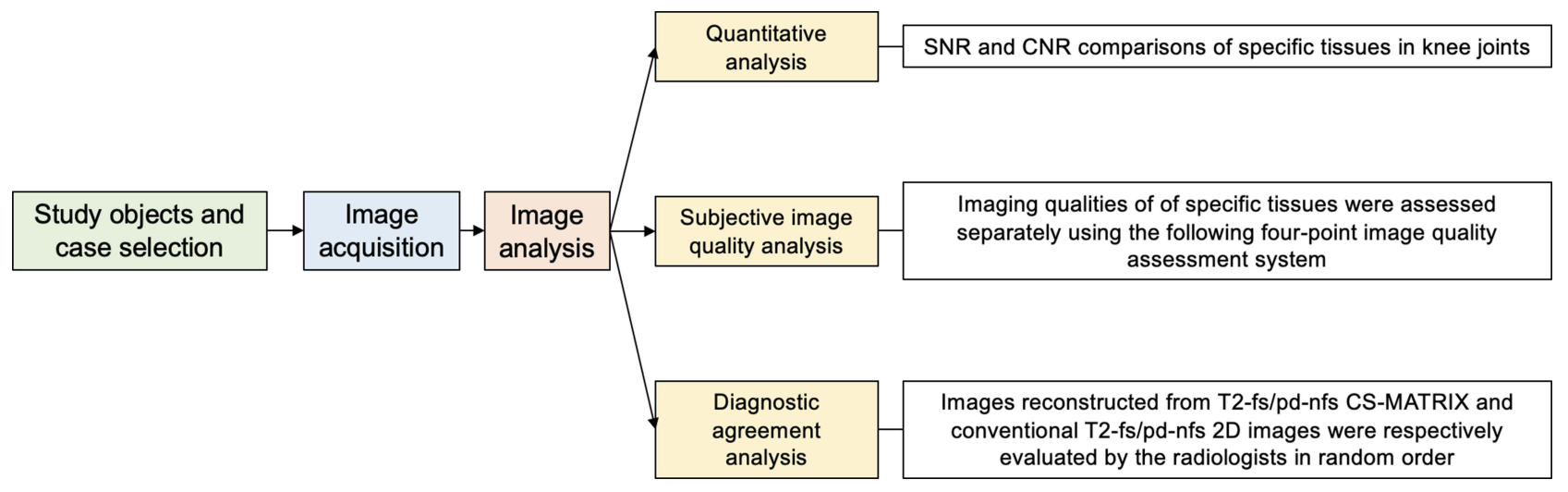

Figure I Experimental flowchart. 


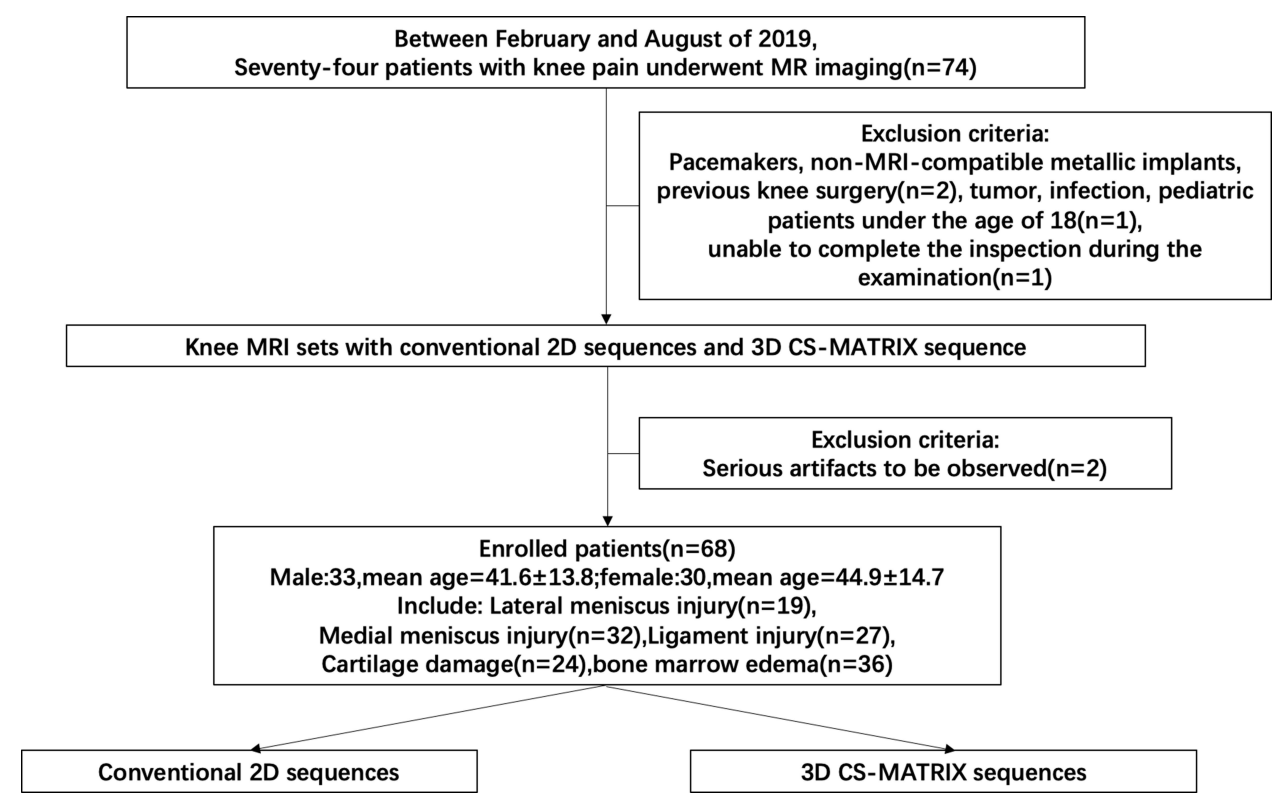

Figure 2 Flowchart of patient enrollment.

age: $41.6 \pm 13.8$ years; female: 30 , mean age: $44.9 \pm 14.7$ years) were screened to be investigated, and clinical treatment was not delayed for any patient because of study participation. The flowchart of patient enrollment is presented in Figure 2.

\section{MRI Protocols}

All patients were examined with a $3.0 \mathrm{~T}$ magnetic resonance (MR) scanner (uMR780; United Imaging Healthcare, Shanghai, China) with a dedicated 12-element transmit/receive (T/R) knee coil. MRI protocols included axial, sagittal, and coronal 2D FSE T2-weighted fat-suppressed (T2-fs) and proton density-weighted non-fat-suppressed (pd-nfs) sequences (FOV, $140 \mathrm{~mm}$; in-plane resolution: $0.5 \times 0.5 \mathrm{~mm}^{2}$; acquisition duration: 15 minutes 30 seconds). CSMATRIX sequences were obtained along the sagittal direction (FOV, $140 \mathrm{~mm}$; spatial resolution: $0.5 \times 0.5 \times 0.5$ $\mathrm{mm}^{3}$; acquisition duration: 11 minutes) with an acceleration factor of five, and the specific parameters of the sequences are shown in Table 1. The post-acquisition axial, sagittal, and coronal MPRs were obtained within the workstation (United Imaging Healthcare, Shanghai, China).

Table I Parameters of MRI Sequences

\begin{tabular}{|c|c|c|c|c|c|c|c|c|}
\hline & $\begin{array}{l}\text { pd-nfs CS- } \\
\text { MATRIX }\end{array}$ & $\begin{array}{c}\text { pd-nfs 2D } \\
\text { Sagittal }\end{array}$ & $\begin{array}{l}\text { pd-nfs 2D } \\
\text { Transverse }\end{array}$ & $\begin{array}{c}\text { pd-nfs 2D } \\
\text { Coronal }\end{array}$ & $\begin{array}{l}\text { T2-fs CS- } \\
\text { MATRIX }\end{array}$ & $\begin{array}{l}\text { T2-fs 2D } \\
\text { Sagittal }\end{array}$ & $\begin{array}{c}\text { T2-fs 2D } \\
\text { Transverse }\end{array}$ & $\begin{array}{l}\text { T2-fs 2D } \\
\text { Coronal }\end{array}$ \\
\hline FOV $\left(\mathrm{mm}^{2}\right)$ & \multicolumn{8}{|c|}{$|40 *| 40$} \\
\hline TR/TE (ms/ms) & $|000 / 7| .4$ & $3122 / 37.7$ & $3176 / 36.8$ & $5080 / 38.5$ & $1400 / 184.2$ & $4876 / 69.1$ & $5317 / 76.7$ & $4562 / 68.8$ \\
\hline Resolution $\left(\mathrm{mm}^{3}\right)$ & $0.5 * 0.5 * 0.5$ & $0.5 * 0.5 * 2.5$ & $0.5 * 0.5 * 3$ & $0.5 * 0.5 * 3$ & $0.5 * 0.5 * 0.5$ & $0.5 * 0.5 * 3$ & $0.5 * 0.5 * 3$ & $0.5 * 0.5 * 3$ \\
\hline FA $\left({ }^{\circ}\right)$ & & 135 & 135 & 135 & & 150 & 120 & 150 \\
\hline ETL & 50 & 14 & 16 & 14 & 80 & 12 & 12 & 12 \\
\hline BW $(\mathrm{Hz})$ & 500 & 200 & 200 & 200 & 400 & 200 & 180 & 200 \\
\hline Slices & 200 & 30 & 30 & 35 & 200 & 25 & 26 & 25 \\
\hline NEX & I & I & I & I & 1.5 & I & I & I \\
\hline TA (min:s) & $4: 41$ & $0: 56$ & $1: 19$ & $2: 32$ & $6: 25$ & $3: 48$ & 4:09 & $2: 53$ \\
\hline
\end{tabular}

Abbreviations: pd-nfs, proton-density-weighted sequence, non-fat-suppressed; CS-MATRIX, three-dimensional modulated flip-angle technique in refocused imaging with an extended echo train and compressed sensing; T2-fs, T2-weighted sequence, fat suppressed; FOV, field of view; TR, repetition time; TE, echo time; FA, flip angle; ETL, echo train length; BW, bandwidth; NEX, number of excitations; TA, acquisition time. 


\section{Image Analysis \\ Quantitative Analysis}

The signal-to-noise ratio (SNR) and contrast-to-noise ratio (CNR) of specific tissues were measured on both conventional 2D and 3D MPR images using the software Horos (https://horosproject.org/). With Horos, regions of interests (ROIs) were manually drawn by musculoskeletal radiologists with 5 years of experience in musculoskeletal imaging. The determination started with the 3D MPR images and then transferred to the 2D images. The reviewers were blinded to electronic medical records, including the results of physical examinations, arthroscopic findings, and diagnoses. Afterwards, the SNRs of cartilage, synovial fluid, fat, bone marrow, ligaments, and menisci were calculated by dividing the average signal intensity $\left(\mathrm{S}_{\text {Tissue }}\right)$ by the standard deviation within the selected ROIs $\left(\sigma_{\text {Tissue }}\right)$, which is regarded as background noise, given that the SNR calculated by the background standard deviation is not uniform across the regions of an accelerated sparse image, namely, $\mathrm{SNR}=\frac{\mathrm{S}_{\text {Tissue }}}{\sigma_{\text {Tissue }}}$. Then, the CNRs of cartilage against synovial fluid, bone marrow, and muscle, and synovial fluid against meniscus, bone marrow, and fat were calculated by $\mathrm{CNR}=\frac{\mathrm{S}_{\text {Tissuel }}-\mathrm{S}_{\text {Tissue } 2}}{\sqrt{\sigma_{\text {Tissue } 1}^{2}+\sigma_{\text {Tissue2 }}^{2}} \cdot 17,19,20}$

\section{Subjective Image Quality Analysis}

Muscoloskeletal radiologists with 5 and 10 years of experience were also enrolled to review the 68 examinations utilizing a four-point grading system to evaluate the image quality independently, according to the clarity of anatomical structures and the presence and severity of susceptibility or blood flow artifacts on the images. The imaging qualities of the medial menisci (MM), lateral menisci (LM), anterior cruciate ligament (ACL), posterior cruciate ligament (PCL), and cartilage-subchondral bone interface were assessed separately using the following four-point image quality assessment system: 4, excellent image quality with sharp delineation of the cartilage from the synovial fluid, clear delineation of the subchondral bone, menisci, and cruciate ligaments, and minimal noise and artifacts; 3, good image quality with less than optimal findings of one or two of the previously mentioned criteria; 2, acceptable image quality, with the diagnostic quality of the images mildly affected by substantial limitations; and 1, poor image quality, with the presence of substantial limitations in the diagnostic quality of the images, extensive artifacts, or noise.

\section{Diagnostic Agreement Analysis}

Images reconstructed from T2-fs CS-MATRIX and conventional T2-fs 2D images were evaluated by the radiologists in random order, and the pd-nfs images were also involved as additional references. The meniscal injuries were assessed depending on the degree of tearing of the anterior/posterior horn $(\mathrm{AH} / \mathrm{PH})$ and the body of the $\mathrm{MM}$ and LM separately. The corresponding diagnostic standards were based on a five-point grading system: 0, no lesion, with uniform low signal all over the meniscus; 1, intra-substance abnormality, with limited dots of increased signal in meniscus; 2, non-displaced tears, increased linear signal within the meniscus and not contacting the meniscal surface; 3 , displaced or complex tears without maceration, with increased intra-substance signal intensity unequivocally contacting the articular surface; and 4, maceration of the meniscus, with meniscus volume reduction or morphological change on the basis of grade. ${ }^{21,22}$ ACL and PCL were evaluated using a three-point grading system: 0, no lesion; 1, partial-thickness tear; and 2, complete tear, which depends on the presence of intra-ligament high signals, abnormal shapes or orientations of ligaments, and discontinuous cruciate ligament signals. ${ }^{23}$ Bone marrow edema (BME) was identified initially on fat-suppressed images by ill-defined (no clear border between normal and abnormal signal intensity) increased signals in subchondral bone, and non-fatsuppressed images by ill-defined low signals. ${ }^{24,25}$ Cartilage damage was diagnosed given the thickness and integrity of the cartilage signal adhering to the bone surfaces, where the linear or focal regions of fluid signals inside usually represent fissures, whereas larger regions indicate cartilage loss, which was commonly observed on pd-nfs MR images. ${ }^{26}$ 
Table 2 SNR and CNR Comparisons of Specific Tissues in Knee Joints

\begin{tabular}{|l|c|c|c|c|c|c|}
\hline & T2-fs CS-MATRIX & T2-fs 2D & P Value & pd-nfs CS-MATRIX & pd-nfs 2D & $P$ Value \\
\hline SNR & & & & & \\
SF & $19.21 \pm 18.50$ & $25.07 \pm 33.75$ & 0.319 & $15.32 \pm 7.41$ & $31.49 \pm 31.32$ & 0.061 \\
BM & $12.75 \pm 4.09$ & $14.24 \pm 4.53$ & 0.229 & $16.67 \pm 6.57$ & $24.55 \pm 3.61$ & 0.004 \\
Cart & $12.31 \pm 6.24$ & $6.58 \pm 2.45$ & 0.001 & $12.24 \pm 5.85$ & $10.02 \pm 6.04$ & 0.187 \\
Meni & $5.45 \pm 1.47$ & $3.09 \pm 1.34$ & 0.001 & $7.51 \pm 2.19$ & $4.62 \pm 1.70$ & 0.002 \\
Liga & $5.74 \pm 2.02$ & $2.60 \pm 1.01$ & 0.032 & $6.17 \pm 2.93$ & $4.17 \pm 1.62$ & 0.044 \\
Fat & $14.63 \pm 5.28$ & $18.33 \pm 7.83$ & 0.169 & $23.60 \pm 9.34$ & $35.70 \pm 20.53$ & 0.028 \\
CNR & & & & & $3.65 \pm 5.09$ & 0.498 \\
Cart vs SF & $8.79 \pm 7.60$ & $8.01 \pm 6.78$ & 0.519 & $2.62 \pm 1.94$ & $7.73 \pm 3.58$ & 0.977 \\
Cart vs BM & $5.23 \pm 3.90$ & $2.88 \pm 1.79$ & 0.013 & $7.71 \pm 1.96$ & $7.02 \pm 3.37$ & 0.975 \\
Cart vs Meni & $9.16 \pm 4.84$ & $5.66 \pm 2.35$ & 0.003 & $7.05 \pm 3.90$ & $10.87 \pm 6.17$ & 0.188 \\
SF vs Meni & $16.33 \pm 14.64$ & $20.15 \pm 23.67$ & 0.312 & $7.07 \pm 4.04$ & $3.15 \pm 2.37$ & 0.000 \\
SF vs BM & $13.97 \pm 12.79$ & $18.41 \pm 22.31$ & 0.252 & $5.14 \pm 2.27$ & $6.70 \pm 2.78$ & $0.18 \pm 2.71$ \\
SF vs Fat & $14.44 \pm 12.96$ & $17.68 \pm 22.12$ & 0.419 & & 006 \\
\hline
\end{tabular}

Abbreviations: SNR, signal-to-noise ratio; CNR, contrast-to-noise ratio; CS-MATRIX, three-dimensional modulated flip-angle technique in refocused imaging with an extended echo train and compressed sensing; pd-nfs, proton-density-weighted sequence, non-fat-suppressed; T2-fs, T2-weighted sequence, fat suppressed; SF, synovial fluid; BM, bone marrow; Cart, cartilage; Meni, menisci; Liga, ligament.

\section{Statistical Analysis}

Quantitative analyses and subjective image quality score comparisons were carried out between the isotropic CSMATRIX and conventional 2D sequences. Individual SNRs and CNRs were quantitatively assessed using the paired $t$-test, and the variations in subjective image quality score between the two sequences were compared using the Wilcoxon signed-rank test. The evaluation of various knee joint pathologies was compared between CS-MATRIX and 2D sequences, using the combination of T2-fs and pd-nfs imaging. The agreement of diagnostic performance was evaluated with Cohen's weighted kappa statistic, and classified as very good (kappa: 0.801-1.000), good (kappa: 0.601-0.800), moderate (kappa: 0.401-0.600), fair (kappa: 0.201-0.400), and poor (kappa: 0.000-0.200). All statistical analyses were performed with IBM SPSS Statistics (IBM SPSS Statistics, version 25.0, Armonk, NY). $P$ values of less than 0.05 indicate statistical significance.

\section{Results}

\section{Quantitative Analysis Results}

The statistical results for the SNRs and CNRs in the selected tissues of CS-MATRIX and conventional 2D sequences are presented in Table 2. Images of T2-fs CS-MATRIX sequences had significantly higher SNRs than those of routine T2-fs 2D sequences in cartilage $(12.31 \pm 6.24$ vs $6.58 \pm 2.45, P=0.001)$, menisci $(5.45 \pm 1.47$ vs $3.09 \pm 1.34, P=0.001)$, and ligaments $(5.74 \pm 2.02$ vs $2.60 \pm 1.01, P=0.032)$, and the CNRs of cartilage against both bone marrow and menisci calculated from T2-fs CS-MATRIX sequences were significantly higher than those of 2D T2-fs sequences $(5.23 \pm 3.90$ vs $2.88 \pm 1.79, P=0.013 ; 9.16 \pm 4.84$ vs $5.66 \pm 2.35, P=0.003)$. As for pd-nfs sequences, the SNRs of menisci and ligaments in CS-MATRIX sequences were also significantly higher than those in conventional 2D sequences $(7.51 \pm 2.19$ vs 4.62 $\pm 1.70, P=0.002 ; 6.17 \pm 2.93$ vs $4.17 \pm 1.62, P=0.044$ ); however, the SNRs of bone marrow and fat were both lower compared with those of $2 \mathrm{D}$ sequences $(16.67 \pm 6.57$ vs $24.55 \pm 3.61, P=0.004 ; 23.60 \pm 9.34$ vs $35.70 \pm 20.53, P=0.028)$. In addition, the CNRs of synovial fluid against bone marrow and fat calculated from the CS-MATRIX sequences were significantly higher than those of the $2 \mathrm{D}$ sequences $(5.14 \pm 2.27$ vs $3.15 \pm 2.37, P=0.000 ; 6.70 \pm 2.78$ vs $4.18 \pm 2.71$, $P=0.006$ ).

\section{Subjective Image Quality Analysis}

The results of image quality comparisons are shown in Figure 3. No significant difference in image quality was found between T2-fs CS-MATRIX and T2-fs $2 \mathrm{D}$ sequences (mean score $=3.35 \pm 0.61$ vs $3.37 \pm 0.59, P=0.876$ ); however, the 


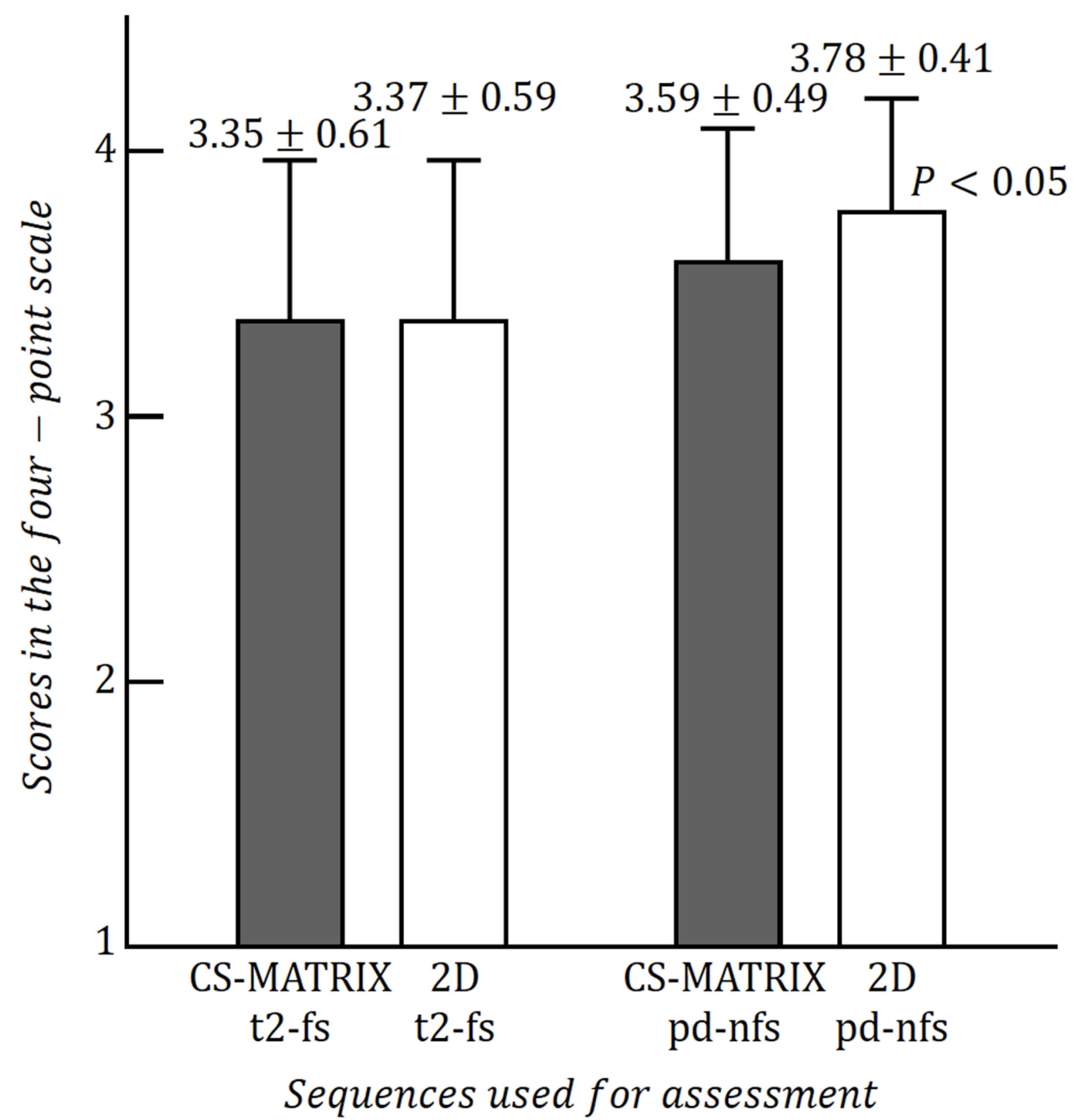

Figure 3 Histograms of the average subjective image quality scores evaluated by the four-point scale for CS-MATRIX T2-fs and 2D T2-fs, and CS-MATRIX pd-nfs and 2D pdnfs sequences. Data show no significant difference between the images obtained from the T2-fs sequences $(P=0.873)$; however, the $2 D$ pd-nfs sequence presents a significantly better image quality than the pd-nfs CS-MATRIX sequence $(P<0.05)$.

image quality scores of routine pd-nfs 2D were significantly higher than those of pd-nfs CS-MATRIX sequence (mean score $=3.78 \pm 0.41$ vs $3.59 \pm 0.49, P=0.007$ ). The CS-MATRIX sequence presents obviously improved image quality in blood flow artifact reduction compared to conventional 2D sequences, as shown in Figure 4.

\section{Diagnostic Agreement Analysis}

The results of diagnostic agreement evaluation are presented in Table 3. There was a very good agreement between the isotropic CS-MATRIX and 2D sequences in detecting cartilage lesions $(\kappa=1.000)$ and BME $(\kappa=0.971)$. However, the range of the edemas shown in images from T2-fs CS-MATRIX sequences was not always as large as that of conventional T2-fs $2 \mathrm{D}$ sequences, and the contrast between edema and surrounding normal bone marrow shown in pd-nfs CS-MATRIX images was better than that of pd-nfs 2D images (Figure 5). The diagnostic agreement reached good to very good in the grading evaluation of MM and LM tears $(\kappa=0.787$, $\kappa=0.910$ ), and two symptomatic cases are shown in Figure 6. More specifically, between the sequences, the AH, body, and PH tears of LM showed very good diagnostic agreement $(\kappa=0.909, \kappa=0.953, \kappa=0.882)$, while the $\mathrm{AH}$, body, and $\mathrm{PH}$ tears of $\mathrm{MM}$ all had moderate to very good diagnostic agreement $(\kappa=0.690 \kappa=0.911$, $\kappa=0.675$ ). Two diagnosed cases of $\mathrm{PH}$ tears of MM with different diagnostic grades are shown in Figure 7. Moreover, the diagnostic agreements for ACL and PCL tears were good to very good and very good, respectively 


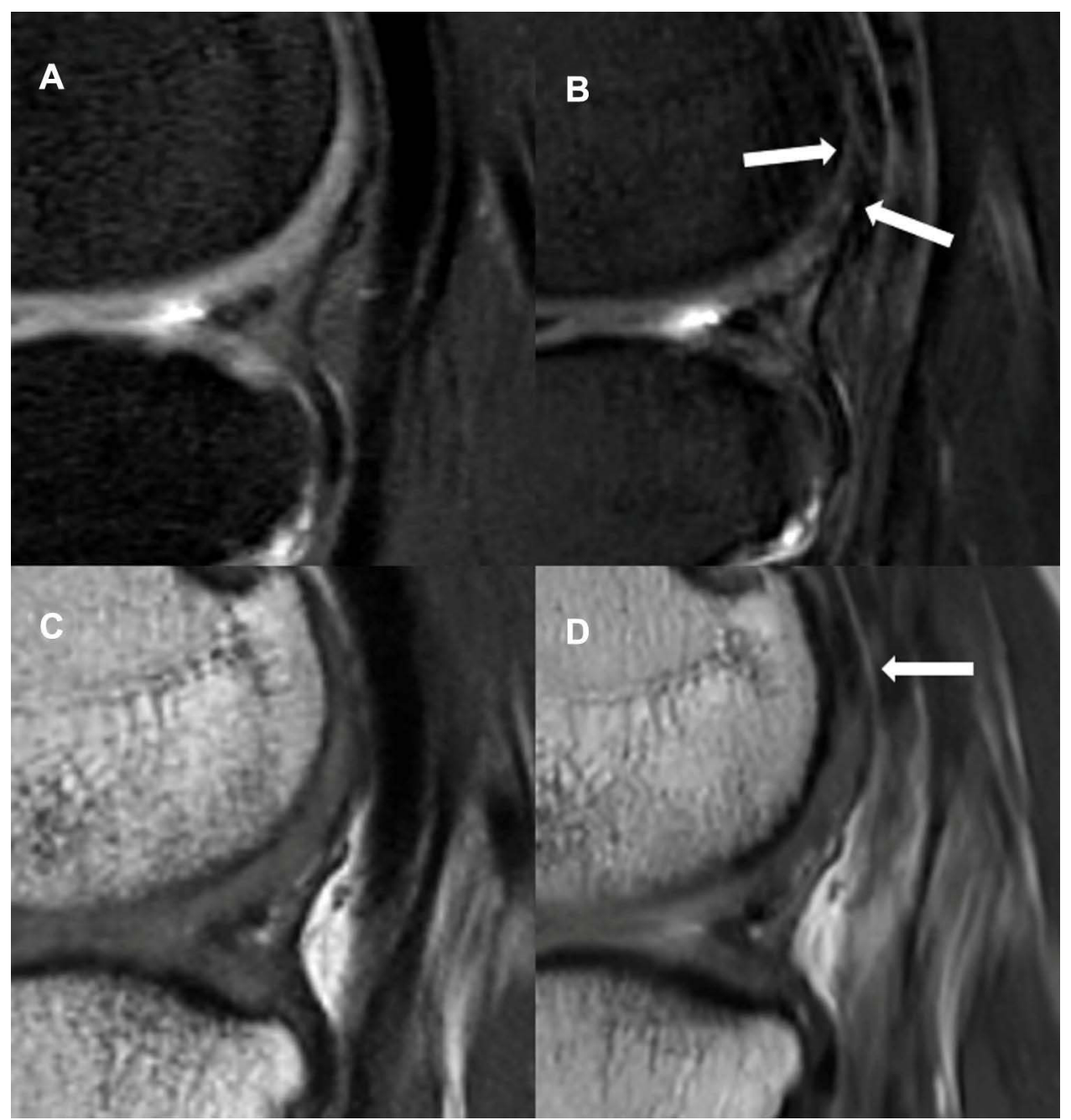

Figure 4 Right knee magnetic resonance images of a 37-year-old man with normal cartilage. The sagittal images reconstructed from T2-fs (A) and pd-nfs (C) CS-MATRIX sequences present a clear delineation of complete cartilage on the lateral condyle, while in the sagittal T2-fs (B) and pd-nfs (D) 2D images, the blood flow artifacts cause obvious signal loss (arrows) on the cartilage, making it difficult to determine whether there is a lesion.

$(\kappa=0.792, \kappa=1.000)$. During diagnosis, CS-MATRIX sequences inherently allow for arbitrary multiplanar reformations to obtain the images in specific angulations, which may be necessary to visualize small knee structures, as shown in Figure 8.

\section{Discussion}

The results of this study demonstrated the successful application of an isotropic 3D variable-flip-angle FSE sequence with CS technique in knee MRI, through various comparisons of CS-MATRIX with conventional 2D sequences. Previous investigations have demonstrated that 3D sequences can improve the diagnostic accuracy compared to conventional 2D sequences in comprehensive evaluations of knee joint injuries. ${ }^{27,28}$ Nonetheless, the isotropic spatial resolution and acquisition time still remain to be optimized in clinical applications. Based on these considerations, the CS technique comes into view. Geethanath et $\mathrm{al}^{14}$ reviewed numerous applications of the CS technique in musculoskeletal system imaging and demonstrated the feasibility of knee imaging with fivefold acceleration. ${ }^{29}$ In this study, with the advantages of both the MATRIX sequence and the CS technique, the accelerated isotropic T2-fs and pd-nfs CS-MATRIX sequences saved about $30 \%$ of total imaging time compared to the conventional $2 \mathrm{D}$ multiplanar sequences. 
Table 3 Diagnostic Agreements of Knee Lesions Diagnosed by CS-MATRIX and Conventional 2D Sequences

\begin{tabular}{|l|c|c|c|}
\hline Tissue for Diagnosis & Kappa Value $(\kappa: P$ Value $(\mathbf{9 5} \% \mathbf{C I}))$ & $\boldsymbol{P}$ Value & Performance \\
\hline LM AH lesion & $0.909 ; 0.049(0.813-1.000)$ & $<0.001$ & Very good \\
LM body lesion & $0.953 ; 0.045(0.865-1.000)$ & $<0.001$ & Very good \\
LM PH lesion & $0.882 ; 0.056(0.772-0.992)$ & $<0.001$ & Good to very good \\
LM lesions & $0.910 ; 0.059(0.85 I-0.969)$ & $<0.001$ & Moderate to very good \\
MM AH lesion & $0.690 ; 0.097(0.500-0.880)$ & $<0.001$ & Good to very good \\
MM body lesion & $0.911 ; 0.062(0.789-1.000)$ & $<0.001$ & Moderate to very good \\
MM PH lesion & $0.675 ; 0.087(0.504-0.846)$ & $<0.001$ & Good to very good \\
MM lesions & $0.787 ; 0.041(0.707-0.867)$ & $<0.001$ & Good to very good \\
All meniscal lesions & $0.845 ; 0.026(0.794-0.896)$ & $<0.001$ & Good to very good \\
ACL lesion & $0.792 ; 0.074(0.647-0.937)$ & $<0.001$ & Very good \\
PCL lesion & $1.000 ; 0.000(1.000-1.000)$ & $<0.001$ & Good to very good \\
All CL lesions & $0.849 ; 0.055(0.74 I-0.957)$ & $<0.001$ & Very good \\
Cartilage lesion & $1.000 ; 0.000(1.000-1.000)$ & $<0.001$ & Very good \\
Bone marrow edema & $0.971 ; 0.029(0.914-1.000)$ & \\
\hline
\end{tabular}

Abbreviations: CS-MATRIX, three-dimensional modulated flip angle in refocused imaging with extended echo trains with compressed sensing; LM, lateral meniscus; $\mathrm{MM}$, medial meniscus; $\mathrm{AH}$, anterior horn; $\mathrm{PH}$, posterior horn; $\mathrm{ACL}$, anterior cruciate ligament; $\mathrm{PCL}$, posterior cruciate ligament; $\mathrm{CL}$, cruciate ligament.

Kijowski et al performed intermediate-weighted 3D FSE sequences (CUBE; GE Healthcare, Waukesha, WI) with CS (CUBE-CS) and without CS (CUBE) on the knees using the same $3 \mathrm{~T}$ scanner, achieving a 30\% reduction in scan time for 3D FSE imaging of the knee without a corresponding decrease in the SNR or diagnostic performance in knee imaging. ${ }^{16}$ The time savings and image qualities in their study are similar to our results. However, the 3D FSE sequences in this study used an anisotropic voxel size of $0.5 \times 0.5 \times 1.0 \mathrm{~mm}$, which degraded the image quality. In their experiment, a single CUBE sequence was used as the comparison object, whereas we selected the routine 2D T2-fs and pd-nfs sequences in the clinic as the comparison objects, which makes the experiment more informative and persuasive. Lee et al compared image quality between CS 3D FSE and conventional 3D FSE sequences for knee MRI. ${ }^{17}$ They only used the 3D FSE sequence for comparison, which cannot highlight the advantages of 3D imaging compared with 2D imaging in diagnosis and image resolution. Altahawi et al compared the image quality and diagnostic performance of accelerated fs 3D FSE sequences with conventional 2D FSE sequences on knee imaging. ${ }^{20}$ In their diagnostic evaluation, the perceived diagnostic values of the tissue were assessed without evaluating the severity of specific lesions within the knee joints, and further studies would be needed for a more comprehensive conclusion. In our study, the results of the diagnostic agreement evaluation revealed that the CS-MATRIX sequences could achieve similar performance to the 2D sequences in evaluating lesions in cartilage, bone marrow, menisci, and cruciate ligaments. This implies that both groups of acquired images presented enough visible contrast of neighboring tissues, resulting from the relative CNRs of both CS-MATRIX and conventional 2D sequences. A previous study demonstrated that the variable-flipangle fs 3D FSE sequence showed significantly lower value than 2D sequences for evaluating bones and ligaments; ${ }^{20}$ however, only the 3D fs sequences were employed for diagnosis. In our study, with the presence of the additional nfs CS-MATRIX sequence, the diagnostic agreement of CL lesions and BME between the CSMATRIX and conventional 2D sequences achieved ratings of good to very good. Additionally, marrow fat is known to present higher signals than edema in pd-nfs images, and lower signals on T2-fs sequence imaging. In clinical diagnosis, the smaller range of BME in images from T2-fs CS-MATRIX sequence may lead to falsenegative diagnoses. In contrast, the low signal in the pd-nfs CS-MATRIX sequence could be mistakenly regarded as edema, which may lead to a false-positive judgment. Compared to the previous results, the combination of fs and nfs CS-MATRIX sequences is considered to have great potential in improving the detection of edema-related pathology, which is consistent with another study. ${ }^{30}$ 


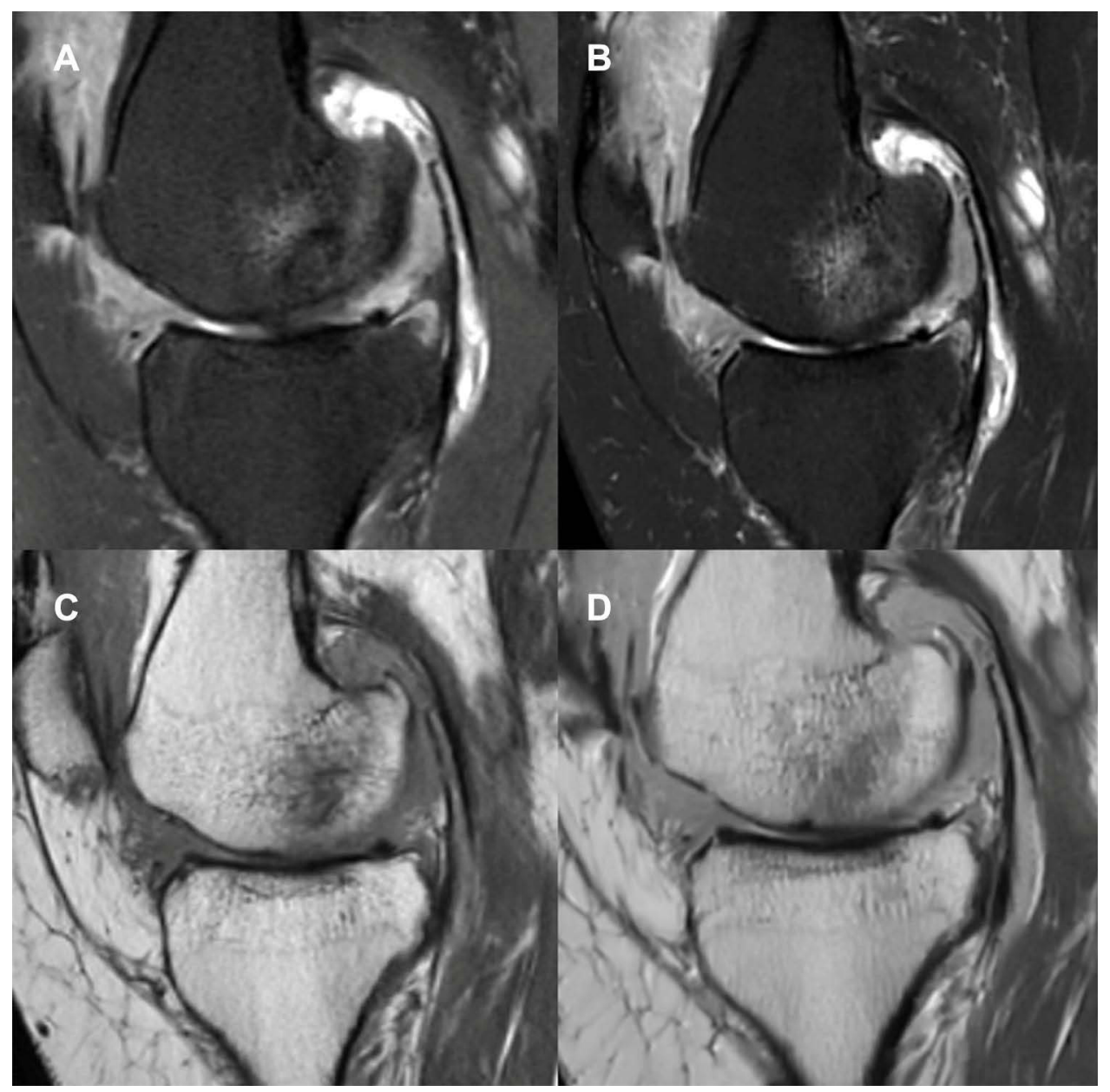

Figure 5 Left knee magnetic resonance images of a 34-year-old man with knee pain. The images show an area of high signals in the bone marrow of the medial condyle on both images obtained from the T2-fs CS-MATRIX (A) and conventional T2-fs 2D sequences (B), and an area of low signal on the image reconstructed from the pd-nfs CSMATRIX sequence (C) and the pd-nfs 2D image (D) at the same anatomical position.

In quantitative analysis, the results indicated that for most tissues the CS-MATRIX sequences could yield a better SNR and CNR than the 2D sequences. 2D sequences constantly show non-isotropic voxels resulting from thick slices, imaging gaps, and partial-volume artifacts that may negatively affect image quality and obscure small pathologies. ${ }^{31}$ In order to overcome these limitations, a decrease in the slice thickness of 2D sequences is required, but thinner slices require a long acquisition time and small voxel size leads to decreased SNR. ${ }^{32}$ According to our results, 3D sequences are more suitable for small voxel acquisition, and the combination of the CS technique with the isotropic 3D variable-flip -angle FSE sequence has provided an acceptable image quality and comparable diagnostic performance on multiple articular pathologies, while reducing the scan time compared with both 3D FSE sequences without CS and conventional 2D sequences. ${ }^{17,20,33,34}$ However, the pd-nfs CS-MATRIX sequence presented lower SNRs in both bone marrow and fat tissue, and this reduction in SNR may give rise to data undersampling in the k-space when utilizing the CS technique with a high turbo factor. ${ }^{14}$ The CS-MATRIX sequences showed superior CNRs in almost every comparison, which can be attributed to the higher signal intensities detected during T2 relaxation by CS-MATRIX sequences. As mentioned, while variable flip angles and shorter ESP are utilized in the sequence, a longer usable duration is found during T2 relaxation, which contributes to the present relatively high signal intensity in the sequence, resulting in stronger contrast and reducing blur effects. ${ }^{18}$ 


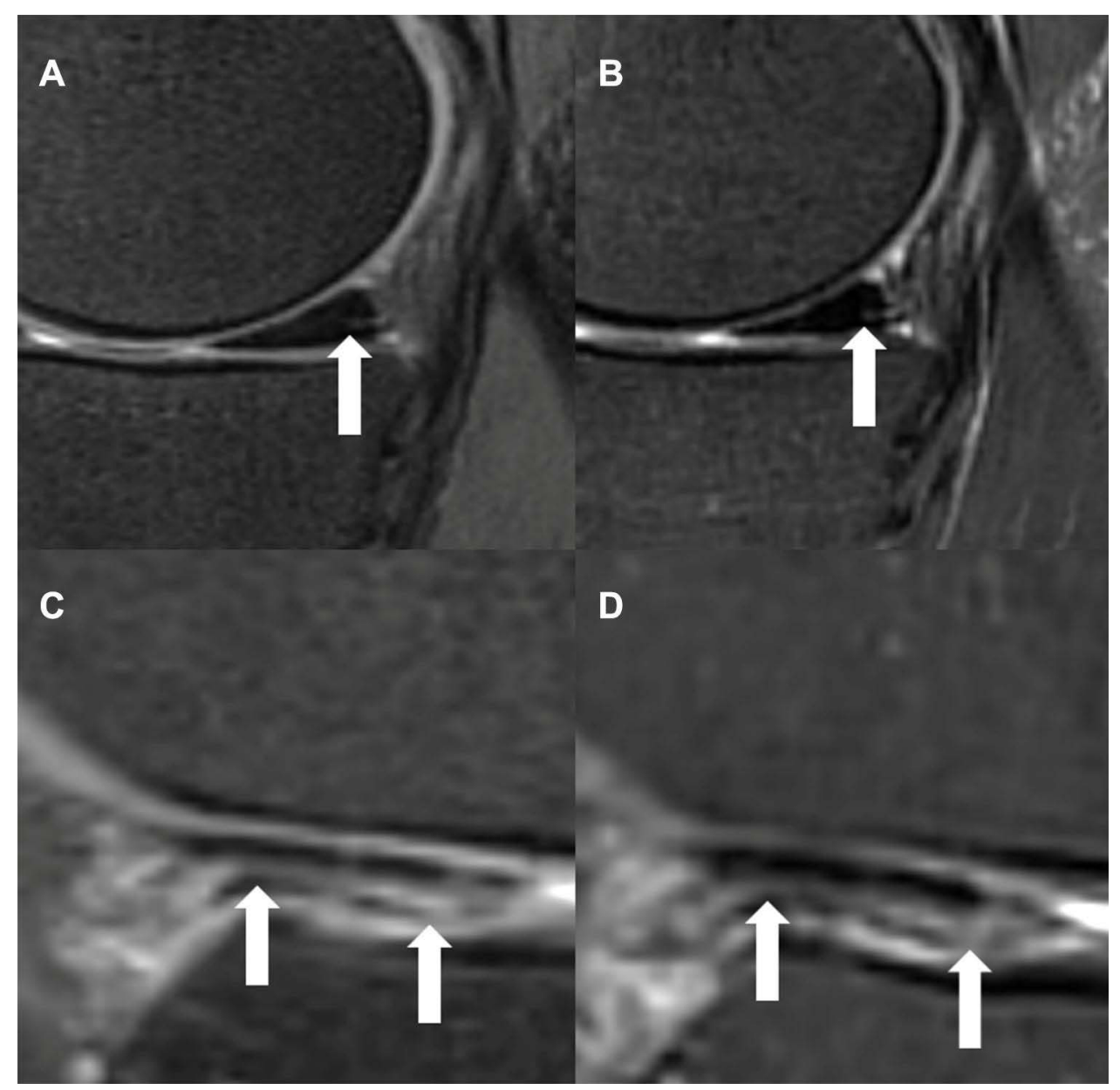

Figure 6 Sagittal images reconstructed from the CS-MATRIX T2-fs sequence (A) and sagittal 2D T2-fs sequence (B) of the left knee of a 47-year-old man with knee pain; from both images the diagnostic results were grade 2 tears on the medial meniscus posterior horn (arrows). Images (C) and (D), respectively, show the sagittal images obtained by CS-MATRIX T2-fs and 2D T2-fs sequences of another symptomatic right knee from a 35-year-old man, and the diagnostic results were grade 3 tears on the lateral meniscus anterior horn (arrows) from both images.

In accordance with our expectations, the CS-MATRIX sequences yielded almost the same image quality as the conventional 2D sequences, even though the average subjective image quality score of the pd-nfs CS-MATRIX sequence was slightly lower (5\%) than that of the pd-nfs 2D sequences. Moreover, CS-MATRIX showed a good reduction in blood flow artifacts, resulting from the completed flow compensation in 3D sequences. With the full flow compensation, which signifies that the flow compensation has been applied in three directions to mitigate the flow artifacts and other negative effects, ${ }^{35,36}$ CS-MATRIX sequences may provide better determination of the images to eliminate fake lesions. Recent advances in MRI have led to the introduction of different 3D techniques aiming to improve both image quality and diagnostic efficiency. This could be attributed to fact that the routinely used 2D sequences always need optimized imaging planes in varying orientations. ${ }^{37,38}$ In contrast, a 3D sequence inherently allows for arbitrary multiplanar reformations to provide clearer insights to visualize small knee structures without extra time consumption. ${ }^{16}$

Unlike a previous study on variable-flip-angle 3D FSE with the CS technique, ${ }^{20}$ lesions within different regions of menisci and cruciate ligaments were evaluated separately in this study, leading to more specific results. Moreover, grading diagnoses of both menisci and cruciate ligament tears were performed in our study to evaluate the practicality of using CS-MATRIX sequences, where patients with different grades of lesions need specialized treatment suggestions. To conclude, our results provide reliable and comprehensive proof of the potential clinical application of this method. 


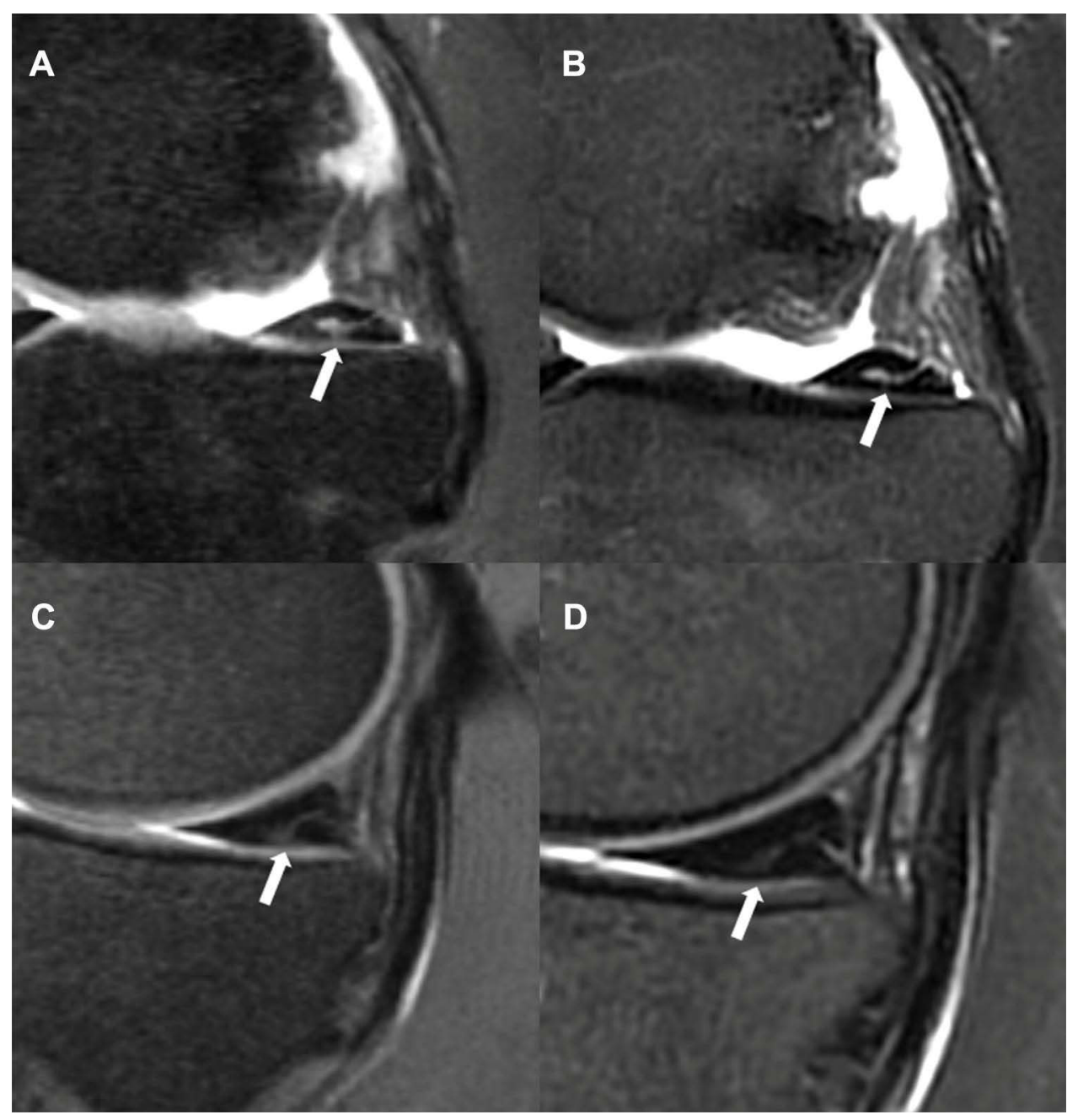

Figure 7 Right and left knee magnetic resonance images of a 46-year-old man with pain in both knees. The T2-fs CS-MATRIX image of the right knee (A) clearly shows a linear high signal (arrow) on the posterior horn of the medial menisci connected to the cartilage surface of the medial condyle; however, the connection (arrow) is almost invisible on the T2-fs 2D image (B). The T2-fs CS-MATRIX image of the left knee (C) also shows a linear high signal (arrow) connected to the cartilage surface of the medial condyle on the posterior horn of the medial menisci; however, the connection (arrow) is also almost invisible on the T2-fs 2D image (D).

\section{Limitations}

There are a few limitations in this study: the sample size was not big and the diagnostic agreement was assessed without involving knee arthroscopies. Studies involving more patients and with examinations under knee arthroscopy or surgery need to be performed to evaluate the diagnostic accuracy directly. Other than that, only different grades of meniscal tears, ligament tears, and cartilage and bone marrow lesions were involved in this study, which did not cover all types of knee lesions seen in the clinic. Therefore, a future study should include more types of knee lesions, so that a more comprehensive conclusion can be made to determine the feasibility of accelerated 3D FSE sequences for clinical application, instead of conventional 2D sequences.

\section{Conclusion}

In conclusion, this study demonstrates the feasibility of employing an isotropic variable-flip-angle FSE sequence with a compressed sensing accelerating technique, CS-MATRIX, in the detection of knee lesions, through analyzing quantitative image quality and subjective image quality, grading the lesions on different parts of the menisci and cruciate ligaments, and evaluating cartilage lesions and BME. The CS technique can provide a $30 \%$ reduction in scan time using 


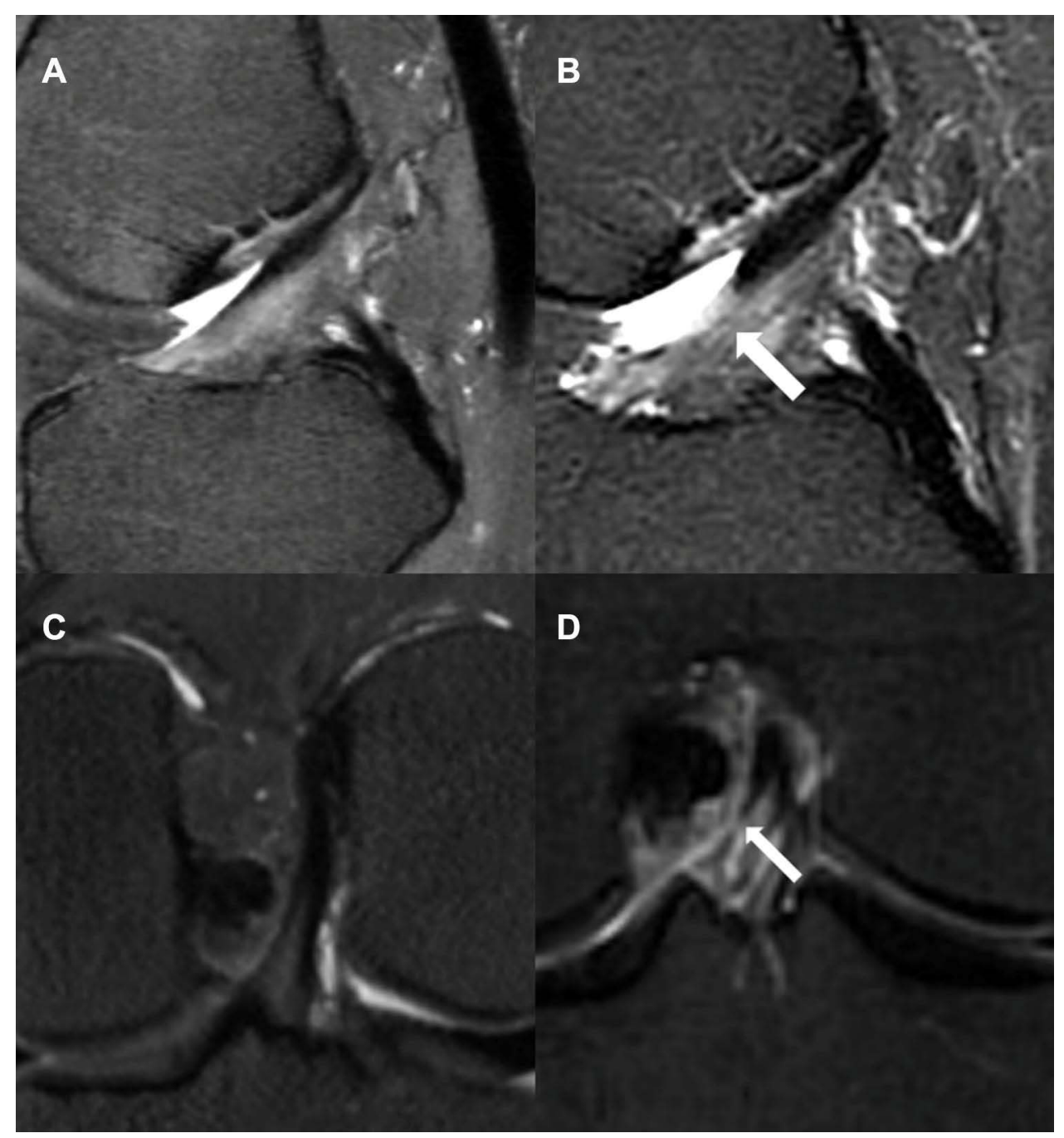

Figure 8 Example magnetic resonance images of knees with normal anterior cruciate ligaments (ACLs). Sagittal images reconstructed from the T2-fs CS-MATRIX sequence (A) show the clear structure of the ACL (arrow); however, the conventional sagittal 2D T2-fs image (B) cannot show the complete ACL in a single slice. In another example with normal ACL, the reformatted oblique coronal image from the T2-fs CS-MATRIX sequence (C) shows the two complete ligament bundles, the anteromedial bundle (AMB) and posterolateral bundle (PLB); however, the conventional coronal 2D T2-fs image (D) can only show the discontinuous AMB (arrow) and unclear PLB.

CS-MATRIX sequences in knee imaging rather than conventional 2D sequences, without a corresponding decrease in image quality or diagnostic performance compared with routine $2 \mathrm{D}$ sequences.

\section{Disclosure}

The authors declare that they have no conflicts of interest for this work.

\section{References}

1. Wadhwa V, Omar H, Coyner K, Khazzam M, Robertson W, Chhabra A. ISAKOS classification of meniscal tears-illustration on 2D and 3D isotropic spin echo MR imaging. Eur J Radiol. 2016;85(1):15-24. doi:10.1016/j.ejrad.2015.10.022

2. Yao L, Pitts JT, Thomasson D. Isotropic 3D fast spin-echo with proton-density-like contrast: a comprehensive approach to musculoskeletal MRI. AJR Am J Roentgenol. 2007;188(2):W199-201. doi:10.2214/AJR.06.0556

3. Oei EH, Nikken JJ, Verstijnen AC, Ginai AZ, Myriam Hunink MG. MR imaging of the menisci and cruciate ligaments: a systematic review. Radiology. 2003;226(3):837-848. doi:10.1148/radiol.2263011892

4. Smith H-J MR. Imaging before arthroscopy in knee joint disorders? Acta Radiol. 2016;37(3P2):601. doi:10.1177/02841851960373p236

5. Kijowski R, Davis KW, Woods MA, Lindstrom MJ, Smet A. Knee joint: comprehensive assessment with 3D isotropic resolution fast spin-echo MR imaging_diagnostic performance compared with that of conventional MR imaging at 3.0T. Radiology. 2009;252(2):486. doi:10.1148/ radiol.2523090028 
6. Subhas N, Kao A, Freire M, Polster JM, Obuchowski NA, Winalski CS. MRI of the knee ligaments and menisci: comparison of isotropic-resolution 3D and conventional 2D fast spin-echo sequences at 3 T. AJR Am J Roentgenol. 2011;197(2):442-450. doi:10.2214/AJR.10.5709

7. Lim D, Han Lee Y, Kim S, Song HT, Suh JS. Clinical value of fat-suppressed 3D volume isotropic spin-echo (Vista) sequence compared to 2D sequence in evaluating internal structures of the knee. Acta Radiol. 2016;57(1):66-73. doi:10.1177/0284185114567560

8. Jee Young J, Young Cheol Y, Jong Won K, Hwan AJ, Bong-Keun C. Diagnosis of internal derangement of the knee at 3.0-T MR imaging: 3D isotropic intermediate-weighted versus 2D sequences. Radiology. 2009;253(3):780-787. doi:10.1148/radiol.2533090457

9. Shakoor D, Kijowski R, Guermazi A, et al. Diagnosis of knee meniscal injuries by using three-dimensional MRI: a systematic review and meta-analysis of diagnostic performance. Radiology. 2019;290(2):435-445. doi:10.1148/radiol.2018181212

10. Chhabra A, Madhuranthakam AJ, Andreisek G. Magnetic resonance neurography: current perspectives and literature review. Eur Radiol. 2018;28 (2):698-707. doi:10.1007/s00330-017-4976-8

11. Madhuranthakam AJ, Lenkinski RE. Technical advancements in MR Neurography. Semin Musculoskelet Radiol. 2015;19(2):86-93. doi:10.1055/ s-0035-1547370

12. Ahlawat S, Carrino JA. Three-dimensional imaging of lower limb neuropathies. Semin Musculoskelet Radiol. 2015;19(2):168-178. doi:10.1055/ s-0035-1546461

13. Naraghi A, White LM. Three-dimensional MRI of the musculoskeletal system. Am J Roentgenol. 2012;199(3):W283-W293. doi:10.2214/ ajr.12.9099

14. Geethanath S, Reddy R, Konar AS, et al. Compressed sensing MRI: a review. Crit Rev Biomed Eng. 2013;41(3):183-204. doi:10.1615/ CritRevBiomedEng.2014008058

15. Lustig M, Donoho D, Pauly JM. Sparse MRI: the application of compressed sensing for rapid MR imaging. Magn Reson Med. 2007;58 (6):1182-1195. doi:10.1002/mrm.21391

16. Kijowski R, Rosas H, Samsonov A, King K, Peters R, Liu F. Knee imaging: rapid three-dimensional fast spin-echo using compressed sensing. J Magn Reson Imaging. 2017;45(6):1712-1722. doi:10.1002/jmri.25507

17. Lee SH, Lee YH, Suh J-S. Accelerating knee MR imaging: compressed sensing in isotropic three-dimensional fast spin-echo sequence. Magn Reson Imaging. 2018;46:90-97. doi:10.1016/j.mri.2017.10.018

18. Mugler JP 3rd. Optimized three-dimensional fast-spin-echo MRI. J Magn Reson Imaging. 2014;39(4):745-767. doi:10.1002/jmri.24542

19. Dietrich O, Raya JG, Reeder SB, Reiser MF, Schoenberg SO. Measurement of signal-to-noise ratios in MR images: influence of multichannel coils, parallel imaging, and reconstruction filters. J Magn Reson Imaging. 2007;26(2):375-385. doi:10.1002/jmri.20969

20. Altahawi FF, Blount KJ, Morley NP, Raithel E, Omar IM. Comparing an accelerated 3D fast spin-echo sequence (CS-SPACE) for knee 3-T magnetic resonance imaging with traditional 3D fast spin-echo (SPACE) and routine 2D sequences. Skeletal Radiol. 2017;46(1):7-15. doi:10.1007/s00256-016-2490-8

21. Ryzewicz M, Peterson B, Siparsky PN, Bartz RL. The diagnosis of meniscus tears: the role of MRI and clinical examination. Clin Orthop Relat Res. 2007;455:123-133. doi:10.1097/BLO.0b013e31802fb9f3

22. Nguyen JC, De Smet AA, Graf BK, Rosas HG. MR imaging-based diagnosis and classification of meniscal tears. Radiographics. 2014;34 (4):981-999. doi:10.1148/rg.344125202

23. Fritz RC. MR imaging of meniscal and cruciate ligament injuries. Magn Reson Imaging Clin N Am. 2003;11(2):283-293. doi:10.1016/s10649689(03)00028-x

24. Zanetti M, Bruder E, Romero J, Hodler J. Bone marrow edema pattern in osteoarthritic knees: correlation between MR imaging and histologic findings. Radiology. 2000;215(3):835-840. doi:10.1148/radiology.215.3.r00jn05835

25. Faruch Bilfeld M, Lapegue F, Brun C, et al. Bone abnormalities of the knee: MRI features. Diagn Interv Imaging. 2016;97(7-8):779-788. doi:10.1016/j.diii.2016.02.011

26. Forney M, Subhas N, Donley B, Winalski CS. MR imaging of the articular cartilage of the knee and ankle. Magn Reson Imaging Clin N Am. 2011;19(2):379-405. doi:10.1016/j.mric.2011.02.005

27. Kudo H, Inaoka T, Kitamura N, et al. Clinical value of routine use of thin-section 3D MRI using 3D FSE sequences with a variable flip angle technique for internal derangements of the knee joint at 3T. Magn Reson Imaging. 2013;31(8):1309-1317. doi:10.1016/j.mri.2013.02.003

28. Ai T, Zhang W, Priddy NK, Li X. Diagnostic performance of CUBE MRI sequences of the knee compared with conventional MRI. Clin Radiol. 2012;67(12):e58-63. doi:10.1016/j.crad.2012.07.020

29. Gold GE, Han E, Stainsby J, Wright G, Brittain J, Beaulieu C. Musculoskeletal MRI at 3.0 T: relaxation times and image contrast. AJR Am $J$ Roentgenol. 2004;183(2):343-351. doi:10.2214/ajr.183.2.1830343

30. Cho HW, Suh JS, Park JO, et al. Three-dimensional fast spin-echo imaging without fat suppression of the knee: diagnostic accuracy comparison to fat-suppressed imaging on 1.5T MRI. Yonsei Med J. 2017;58(6):1186-1194. doi:10.3349/ymj.2017.58.6.1186

31. Kijowski R, Davis KW, Woods MA, et al. Knee joint: comprehensive assessment with 3D isotropic resolution fast spin-echo MR imagingdiagnostic performance compared with that of conventional MR imaging at 3.0 T. Radiology. 2009;252(2):486-495. doi:10.1148/ radiol.2523090028

32. Rajeswaran G, Lee JC, Healy JC. MRI of the popliteofibular ligament: isotropic 3D WE-DESS versus coronal oblique fat-suppressed T2W MRI. Skeletal Radiol. 2007;36(12):1141-1146. doi:10.1007/s00256-007-0385-4

33. Rehnitz C, Klaan B, von Stillfried F, et al. Vergleich moderner 3D- und 2D-MR-sequenzen zur bildgebung der hand bei 3 Tesla. [Comparison of modern 3D and 2D MR imaging sequences of the wrist at 3 Tesla]. Rofo. 2016;188(8):753-762. doi:10.1055/s-0042-104512

34. Lee SH, Lee YH, Song HT, Suh JS. Rapid acquisition of magnetic resonance imaging of the shoulder using three-dimensional fast spin echo sequence with compressed sensing. Magn Reson Imaging. 2017;42(1):152-157. doi:10.1016/j.mri.2017.07.022

35. Deistung A, Dittrich E, Sedlacik J, Rauscher A, Reichenbach JR. ToF-SWI: simultaneous time of flight and fully flow compensated susceptibility weighted imaging. J Magn Reson Imaging. 2009;29(6):1478-1484. doi:10.1002/jmri.21673

36. Wu D, Liu S, Buch S, Ye Y, Dai Y, Haacke EM. A fully flow-compensated multiecho susceptibility-weighted imaging sequence: the effects of acceleration and background field on flow compensation. Magn Reson Med. 2016;76(2):478-489. doi:10.1002/mrm.25878 
37. Kijowski R, Blankenbaker DG, Klaers JL, Shinki K, De Smet AA, Block WF. Vastly undersampled isotropic projection steady-state free precession imaging of the knee: diagnostic performance compared with conventional MR. Radiology. 2009;251(1):185-194. doi:10.1148/radiol.2511081133

38. Notohamiprodjo M, Horng A, Pietschmann MF, et al. MRI of the knee at 3T: first clinical results with an isotropic PDfs-weighted 3D-TSEsequence. Invest Radiol. 2009;44(9):585-597. doi:10.1097/RLI.0b013e3181b4c1a1

Journal of Pain Research

\section{Dovepress}

\section{Publish your work in this journal}

The Journal of Pain Research is an international, peer reviewed, open access, online journal that welcomes laboratory and clinical findings in the fields of pain research and the prevention and management of pain. Original research, reviews, symposium reports, hypothesis formation and commentaries are all considered for publication. The manuscript management system is completely online and includes a very quick and fair peer-review system, which is all easy to use. Visit http://www.dovepress.com/testimonials.php to read real quotes from published authors.

Submit your manuscript here: https://www.dovepress.com/journal-of-pain-research-journal 